\title{
Themes, events, and episodes in autobiographical memory
}

\author{
CHRISTOPHER D. B. BURT and SIMON KEMP \\ University of Canterbury, Christchurch, New Zealand \\ and \\ MARTIN A. CONWAY \\ University of Durham, Durham, England
}

\begin{abstract}
The process by which experience is divided into events was examined. Experiment 1 involved diarists recording their experiences over a 3-month period. Diary entries were later transcribed onto cards and the diarists arranged their cards so as to define events they had experienced, and in a separate phase arranged their cards so as to describe the themes that reflected their life. Examination of event- and theme-building strategies indicated that boundaries were frequently used, and events and themes were often formed from clusters of experience combined using content association rather than temporal sequence. Experiment 2 involved photographs taken by the participants, employed the same procedures as in Experiment 1, and revealed event- and theme-building strategiessimilar to those identified in Experiment 1. In Experiment 3 the size of the stimulus set from which events and themes were constructed was manipulated; this did not influence construction strategies. Overall, the experiments show that both autobiographical events and themes frequently consist of episodes taken from more than 1 day.
\end{abstract}

In the study of human memory, the terms episode and event, and more recently theme, are frequently used to refer to knowledge corresponding to autobiographical memory. Although such terms define key structures in theory, their psychological reality has rarely been researched, and their existence, content, and structure are, instead, often presupposed (but see Wheeler, Stuss, \& Tulving, 1997). In this article we report new research that examines the nature of episodes, events, and themes in autobiographical memory. We show how these concepts refer to ways of organizing autobiographical knowledge that people actually use, and we integrate our findings with a recent model of autobiographicalmemory (Conway \& Pleydell-Pearce, 2000) as we try to answer the question: What is an "event" in autobiographical memory?

A large number of diary studies, in which diarists record one or more autobiographical events each day for some period, can be found in the literature (e.g., Burt, 1992; Conway, Collins, Gathercole, \& Anderson, 1996; Linton, 1975; Wagenaar, 1986; White, 1982). Arguably, the diary method has primarily involved collecting specific on-one-day events as the dominant experimental stimuli (e.g., a visit to the beach, lunch with a colleague) and assumed that these are autobiographical events.

This research was supported by a grant from the Marsden Fund (MS1012). We thank Colin MacLeod, David Rubin, Harvey Marmurek, and an anonymous reviewer for commenting on earlier versions of this manuscript. Correspondence should be addressed to C. D. B. Burt, Department of Psychology, University of Canterbury, Private Bag 4800, Christchurch, New Zealand (e-mail: c.burt@psyc.canterbury.ac.nz).
However, are such entries really autobiographical events, or could they be fragments or components of events? The importance of this question is increased if we compare typical diary entries with data from a study by Barsalou (1988), who asked college students to recall events occurring over the previous summer. He found reports of specific on-one-day memories to be comparatively few, whereas reports of more extended or general events were frequent. These "events" were larger, in the sense of covering a longer period, than those generally recorded by diarists, and in consequence they were also more complex.

This broad spectrum of events differing in duration and complexity and referring to highly specific unique experiences as well as to extended and repeated experiences has been termed general events (Conway, 1996). The evidence indicates that general events act as organizing representations for memories of more specific occurrences, as well as providing access to abstract or thematic personal knowledge (Conway \& Pleydell-Pearce, 2000). Yet little is known of this class of events, although they seem to constitute a central feature in autobiographical memory. They seem to be the preferred level of entry (Haque \& Conway, 2001), the level at which information is optimized as neither too specific nor too abstract, and the level that preserves important information about goal attainment (see Barsalou, 1988; Conway \& PleydellPearce, 2000; Robinson, 1992). From the present perspective, it seems that general events might have a psychological reality both in terms of how knowledge is represented in autobiographical memory and how people access and construct memories. 
In three experiments, participants classified either diary entries (Experiment 1) or photographs (Experiments 2 and 3 ) as events. An objective of the present work was to determine the extent to which on-one-day experiences (the typical diary entry) would be classified as events by the people who experienced them, and the extent to which these, if not classified as an event in their own right, would be clustered together to form more elaborate general event representations. On the reasoning that most experiences have associated components, rather than occurring in isolation from other experiences, we predicted that clustering of experiences to form larger, more complex general event structures would dominate the data. Specifically, experiences lead to other associated experiences and are linked by general associative elements such as goals (e.g., the shopping and celebrating events occurring at Christmas), relationships (e.g., meeting someone, going on a first date, etc.), or projects (buying sporting gear, planning a trip, engaging in the sport, etc.), and we predict that experiences are organized in memory in a similar way. From an experimental perspective, our work may suggest a reconsideration of how researchers define their autobiographical event stimuli.

In each experiment, participants also identified life themes present in their recorded experiences. Autobiographical memory theory (see Conway, 1996; Conway \& Pleydell-Pearce, 2000) suggests that the most abstract level of autobiographical knowledge is the themes or lifetime periods that characterize an individual's life (e.g., a relationship with $\mathrm{X}$ ). General events are suggested to be associated with life themes, but the precise nature of the linkages has received little research attention. Here we examined event and theme structures using the same sample of stimuli, and this allowed us to identify the interrelations between events and themes. It was predicted that theme structures would be constructed using components from multiple events, and that fewer themes than events would be identified. Fewer themes than events were expected simply because at any one point in time most individuals' lives are defined by a relatively small number of themes: We generally engage only in one close relation ship at a time, participate in one or two sports, have a limited number of hobbies, and so on. In Experiments 1 and 2, ratings of boundary event/theme components, and event/theme components within boundaries, were also collected to gauge the clarity of boundary event/theme components within the behavioral sequence.

The process by which events and themes are defined was of particular interest. If diary entries (Experiment 1 ) and photographs (Experiments 2 and 3 ) are found to be clustered to form general events and to represent life themes, it is possible to examine the characteristics of the clustering process. As extremes, there appear to be two possibilities: Clusters can be formed from temporally continuous diary entries/photographs or they can be formed by content-associated items. Although experience is obviously continuous, individuals' lives are often characterized by numerous activities that run in parallel.
For example, social events are planned and occur over time, relationships develop over time, projects, such as research and writing this article, occur over time, and in each example there are other activities happening in parallel. Previous research on temporal memory argues against event memory being based around temporal structures. Generally, individuals show very little ability in retrieving temporal information for events and event components (e.g., Burt, Kemp, Grady, \& Conway, 2000; Burt, Watt, Mitchell, \& Conway, 1998). The general conclusion from research on temporal memory is that there is little empirical support for the general use of time tagging for autobiographical events (see Friedman, 1993, for a review of dating research), an exception being the time tags associated with landmark events (see Shum, 1998). Thus our prediction was that content association, rather than temporal association, would be used to form general events and themes.

In summary, we conducted three experiments to address the fundamental issue of what defines an autobiographical event. We collected stimuli using either diary entries (Experiment 1) or photographs (Experiments 2 and 3) and asked the individuals who supplied the stimuli to sort them on the basis of the events and themes in their lives that the stimuli defined. Our procedures allowed for the examination of several predictions relating to how memorial structure would influence the sorting tasks. In particular, the complex interrelations between events and life themes were examined with the expectation of contributing to our understanding of the hierarchical associative structures that constitute autobiographical memory.

\section{EXPERIMENT 1}

\section{Method}

Participants. Fourteen female students aged $18-39$ years $(M=$ 23.4 years) kept diaries over the summer.

Diary keeping. Participants were supplied with a diary to keep over the summer vacation-November 1 to February 28 (120 days). Participants were paid for each entry, at a rate of 20 cents each, up to a maximum of four per day. They were instructed that a complete entry must include what happened, where it happened, and with whom, and they were provided with examples (e.g., Bill and I had a picnic at Sandy Beach). Participants also rated the importance of each recorded experience using a 5-point scale $(1=$ not important; $5=$ extremely important $)$.

Stimulus construction. The researchers selected entries from the diaries using the following criteria: (1) only one entry from each day (where possible) was selected, this being the most unique (as determined after the researchers had read all the entries), and (2) the entry had to have the level of specificity required by the instructions. Each selected entry was typed onto a card. Retention interval was calculated from the date of the diary entry to the day the participant completed the experiment and ranged from 80 to 219 days ( $M=155$ days).

Procedure. The procedure involved two phases. Task order was counterbalanced, with half the participants completing Phase 1 before completing Phase 2 and the other half completing Phase 2 before completing Phase 1. In Phase 1, the participant was presented with her entries in a random order and instructed to "please arrange the cards so as to represent the events you experienced over the summer." Participants were informed that what constituted an event was 
entirely up to them. The experimenters specifically avoided any reference to clustering entries or forming piles. A large table was provided for the task with ample space for the participant to lay out all of her cards individually if she so wished. There was no time limit for the task. Next, the participant wrote a brief label for each event that she had identified and rated each event according to how hard/easy it was to decide that the diary entries belonged to the event on a 7-point scale ( $1=$ extremely hard; 7 = extremely easy), and where more than two entries had been arranged into an event, to rate how hard/easy it was to define the boundaries of the event on a 7 -point scale $(1=$ extremely hard $; 7=$ extremely easy $)$. These scales allowed us to determine whether the initiating and terminating components of events (the boundary points) were more readily assignable to an event than were the components that filled the event.

In Phase 2, the participant was given her entries in a random order and instructed "to arrange them so as to describe themes in your life." There was no time limit. Next the participant wrote a brief label for each theme constructed, rated each theme on how hard/easy it was to form, and where more than two entries were used to define a theme, rated how hard/easy it was to define the boundaries, using the same scales as for Phase 1 .

\section{Results and Discussion}

Event and theme construction. In Experiment 1, there was a total of 1,255 diary entries, ranging from 42 to $119(M=89.6)$ per participant. In previous studies, each entry would be assumed to represent an event, but our participants reduced this total to 249 events by combining multiple diary entries. Of the 249 events, 74 were constructed from a single diary entry $(5.8 \%$ of the diary entries or $29.7 \%$ of the events). Thus it was relatively rare for a single diary entry to be considered an event by the participants. The top half of Table 1 shows the average event and theme construction results from Experiment 1 . As expected, the total number of themes identified by the participants $(n=131)$ was smaller than the total number of events. The number of diary entries used in the construction of events and themes (their size) was averaged for each participant and the means compared. The size of themes $(M=10.96)$ was on average significantly larger than the size of events $(M=6.45)[F(1,13)=$ $\left.12.74, M S_{\mathrm{e}}=11.16, p<.01\right]$.
The use of multiple diary entries to form over $70 \%$ of the events allowed for the isolation of boundary entries. The participants provided entries for a specific reference period; thus some event boundaries (those near the beginning and end of the reference period) may not have been sampled. On the other hand, we did sample from the participants' entire summer vacation, which in itself has marked boundaries, thus increasing the chances of capturing event boundaries. However, it is possible that some entries that we classify as boundaries may in fact only be the first or last part of an event that was available from the sample of entries. The simple implication of this is that any factors we identify for boundary entries are likely to be an underestimation. Participants' ratings of the difficulty of defining the boundaries of each event and theme, and their ratings of how easy it was to decide that an entry belonged to an event/theme, were averaged for each participant (separately for events and themes).

Table 1 shows the overall results. The mean rating for the difficulty of finding boundaries for themes was significantly larger than the corresponding mean for events $\left[F(1,13)=8.17, M S_{\mathrm{e}}=0.13, p<.01\right]$, showing that it was relatively harder to determine the boundaries of an event than to determine the boundaries for a life theme, although both were rated easy (recall that $7=$ extremely easy). There was no significant difference in mean belonging ratings for themes and events, both of which were at the easy end of the scale $\left[F(1,13)=0.97, M S_{\mathrm{e}}=\right.$ 0.17 , n.s.]. Comparison within the events indicated that the mean boundary ratings were significantly smaller than the mean belonging ratings $\left[F(1,13)=4.22, M S_{\mathrm{e}}=\right.$ $0.10, p<.05$ ], showing that it was easier to tell whether an entry belonged in an event than it was to define the event's boundaries. A similar comparison for the themes indicated no significant difference $[F(1,13)=0.02$, $M S_{\mathrm{e}}=0.13$, n.s.].

Event and theme structure. The multiple-entry events and themes could have been constructed either by combining diary entries that described temporally continuous experiences or by combining entries that were asso-

Table 1

Participant-Defined Events and Themes, and Ratings for Event and Theme Construction

\begin{tabular}{|c|c|c|c|}
\hline & Mean Number & $\begin{array}{l}\text { Mean Boundary } \\
\text { Difficulty Rating }\end{array}$ & $\begin{array}{c}\text { Mean } \\
\text { Belonging Rating } \\
\end{array}$ \\
\hline Event and Theme Type & Per Participant & $S D$ & $S D$ \\
\hline
\end{tabular}

Experiment 1

Recorded diary entries

89.6

Participant-constructed single diary entry events

Participant-constructed multiple diary entry events

Participant-identified themes

89.6
5.2
17.7

17.7

9.3

Experiment 2

99

5.8

20.6

7.7

$\begin{array}{llll}5.4 & 1.4 & 5.7 & 1.2 \\ 5.8 & 1.5 & 5.9 & 1.3\end{array}$

1.3

Photographs taken

Participant-constructed single-photograph events

Participant-constructed multiple-photograph events

Participant-identified themes

Note-Ratings for boundary difficulty and belonging ranged from 1 (extremely hard) to 7 (extremely easy). 
ciated by their content. If temporal contiguity was used, there should be a strong association between the number of diary entries in an event or theme and the number of days covered by the event or theme. In contrast, if, as we predicted, content association was used, then there should be no systematic relation between the number of entries in an event or theme and the period of time covered. A boundary span score, equaling the number of days between the earliest and latest diary entries, was calculated for event and theme sizes where there were at least 10 cases across participants. Thus, for events with two, three, four, five, and six diary entries $(65.7 \%$ of all events), the mean boundary spans were $23.1,22.2,21.4$, 45.5, and 52.4 days, respectively, showing that close temporal contiguity was generally not used to form events. Similarly, three theme sizes had at least 10 cases across participants (Theme Sizes 3, 4, and 6, representing $28.6 \%$ of the themes) and produced mean boundary spans of 43.1, 47.2, and 50.6 days, respectively. Thus, for those themes that could be analyzed in this way (note that themes were more variable in length than were events), content association appears to be the preferred form of organization.

We calculated what the average estimated minimum boundary spans would have been if the participants had used temporal contiguity by dividing 120 (the number of days over which diary records were kept) by the number of entries the participant sorted $(M=89.6)$. These average between-diary-entry durations were averaged over the participants; the minimum estimated boundary span for events/themes with two entries was 1.48 (any two entries had to be on average separated by a minimum of 1.48 days). To obtain the estimated minimum boundary spans for other event/theme sizes, the latter value was multiplied by $n-1$ of the number of entries in the event/ theme; thus, estimated minimum boundary spans for event/themes with sizes of three, four, five, and six were 2.96, 4.44, 5.92, and 7.4, respectively. Comparison of these values with the actual boundary spans that we found supported our conclusion that content association was the preferred form of organization.

Event and theme interrelations. The participants' identification of both events and themes from the same sample of diary entries allowed for examination of how events and themes were interrelated. The 74 events constructed from a single entry could, of course, be assigned to only one theme each. Examination of the remaining 175 events showed that for $78(46 \%)$ of these events, component experiences were assigned to one theme, for $56(32 \%)$, component experiences were associated with two themes, and for the remaining 41 (22\%), component experiences were associated with between 3 and 11 themes. Thus, in total, 152 events were associated with a single theme ( $61 \%$ of the total corpus), and the remainder were associated with multiple themes. Of the 131 themes, $16(12 \%)$ were constructed of components from one event only, 53 (40\%) were constructed of components from between 2 and 6 events, and $62(48 \%)$ were constructed of components from between 7 and 41 events. Taken together, these data show that events can be associated with single or multiple themes, with a bias toward single-theme associations, whereas themes tend to be associated with more than one event.

Event and theme boundaries. A total of 350 entries were classified as boundaries in the 175 events constructed from more than 1 entry. Because the participants used the same entries to construct both events and themes, it was possible to determine how many of the entries were used as boundaries for both events and themes. In total, 127 entries were identified as both event and theme boundaries, 67 defining a remote boundary and 57 a recent boundary ( 2 entries defined recent boundaries for events and remote boundaries for themes, and 1 entry defined a remote event but a recent theme boundary).

To explore the nature of event- and theme-delineating boundary experiences, the 1,255 entries were divided into four groups: single entries used to define events $(n=74)$, entries used to define remote boundaries $(n=$ $175)$, entries used to define recent boundaries $(n=175)$, and entries captured within event boundaries $(n=831)$. Mean importance ratings were calculated for each of the four entry types for each participant, and these means were compared. Only 10 participants had data in each of the four entry types (4 participants constructed no events from a single entry). Single-entry events, remote boundary entries, recent boundary entries, and middle entries produced overall importance rating means of 3.1, 2.7, 2.9 , and 2.7 , respectively. Whereas single-entry events were rated on average as more important, the latter means did not differ significantly $[F(3,27)=2.37$, $\left.M S_{\mathrm{e}}=0.15, p=.09\right]$.

Experiment 1 provides evidence that autobiographical events can be complex associations of temporally disparate episodes. These results address the nature of the stimuli we should use to study autobiographical event memory: Studies examining single on-one-day diary entries are perhaps only examining autobiographical episodes. Our design allowed for an examination of the associations between events and themes, and our results suggest that complex hierarchical structures, with multiple nested structures, can be identified. This is not a new idea; it has been proposed by Neisser $(1986,1988)$ as a feature of autobiographical memory organization.

\section{EXPERIMENT 2}

Photographs were used as the stimuli in Experiment 2 to allow an examination of event and theme formation at a different level of resolution. Our experience with photographic archive generation (e.g., Burt, Mitchell, Raggatt, Jones, \& Cowan, 1995; Burt et al., 1998) indicates that photographs are often taken in clusters and provide a rich representation of the components within specific behavioral sequences (e.g., a participant might take 10 photographs at a picnic whereas she might only write a single diary entry describing the experience). A question 
explored in this experiment was whether increasing the level of resolution used to sample behavior would influence participants' classification of stimuli into events.

\section{Method}

Participants. Twenty-seven students who each had access to a camera, 18 females aged $19-49$ years $(M=21.3)$ and 9 males aged 20-39 years $(M=23.2)$, participated in return for their developed photographs.

Procedure. Participants were given three rolls of 36-exposure color film to use over their summer vacation (November 1 to February 28). Participants were instructed (1) to photograph their activities in the way they "normally" would, (2) to take each photograph themselves, (3) to turn off any date-printing functions on their camera, and (4) to return the film unprocessed. Each roll of film was labeled and the participants recorded the date they loaded the film into their camera. This information, and the film negatives, identified the actual temporal order for each photograph. Film was developed into $16 \times 10.5 \mathrm{~cm}$ prints. Prints that were out of focus or badly exposed were removed. All other photographs were used with no restriction on the number of photographs from a single day. An approximate retention interval measure was calculated for each participant using the date that the last roll of film was loaded into the camera and the date on which the participant completed the experiment. This ranged from 80 to 170 days ( $M=124$ days). In the test phases, which were identical to those used in Experiment 1, Phase 1 and Phase 2 were counterbalanced across participants.

\section{Results and Discussion}

Event and theme construction. A corpus of 2,678 usable photographs was collected (a mean of 99 photographs per participant, range 55 to 118). From this corpus, participants constructed 558 events, 159 of which were defined by a single photograph each (6\% of photographs and $28 \%$ of events). A total of 208 life themes were identified. The lower half of Table 1 shows the mean event and theme construction results. Event and theme size (measured by the number of photographs used in their construction) were averaged separately for each participant, and the means were compared. As in Experiment 1, themes were constructed of a significantly larger number of photographs (mean size $=14.74$ ) than were events $($ mean size $=5.62)\left[F(1,26)=83.30, M S_{\mathrm{e}}=\right.$ $13.47, p<.01]$. Participants' ratings of the difficulty of defining the boundaries of each event/theme, and their ratings of how easy it was to determine that photographs belonged to an event/theme, were averaged using only events/themes with a size greater than 1. Comparison of mean ratings of how difficult it was to form the boundary for themes and events (Table 1) revealed a significant difference $\left[F(1,26)=4.83, M S_{\mathrm{e}}=0.50, p<.05\right]$. There was no significant difference in belonging ratings. Comparisons within events between mean boundary and mean belonging ratings revealed a difference that approached significance $\left[F(1,26)=4.13, M S_{\mathrm{e}}=0.08, p=\right.$ $.052]$, and for the themes this difference was significant $\left[F(1,26)=11.77, M S_{\mathrm{e}}=0.16, p<.01\right]$. These results suggest that photographs used to define boundaries were somewhat less strongly associated with the boundary than were photographs contained within the event or theme structure.
Event and theme structure. Multiple-photograph events and themes were examined to determine whether their construction resulted from temporal grouping or content association. Boundary span was calculated using the number of photographs taken between the temporally first and last photographs assigned to an event or theme (but only for event and theme sizes where there were at least 10 cases across participants). For Event Sizes 2-12 (66\% of events), mean boundary spans were $2.9,7.2,8.5$, $13.9,20.5,15.5,20.4,20.4,16.5,17.3,18.3$, and 25.8 , respectively. For Theme Sizes $2-8$ (47\% of themes), the mean boundary spans were 51.6, 26.5, 34.8, 34.7, 16.9, 36.8 , and 41.6, respectively. As in Experiment 1, the photographs used to construct events/themes appear to be combined by content association rather than temporal order. As noted, in this experiment some days were represented by more than one photograph in the stimulus set, and this precluded the calculation of minimum boundary spans. However, this situation, in conjunction with the results we obtained, strongly argues against the value of temporal contiguity as a useful organizing principle for events and themes.

The relation between events and themes was also examined. For multiple-photograph events, all the photographs of a given event were assigned to a single theme in $57 \%$ of cases; in the remainder of events, the photographs were associated with multiple themes. Examination of the number of different events from which components were used to build each theme revealed that only $4 \%$ of themes were defined by a single event; the rest consisted of multiple-event components, with over $36 \%$ of themes consisting of components from more than 10 events.

Boundary characteristics. The 2,678 photographs were divided into four groups: single photographs used to define events $(n=159)$, remote-boundary or initiatingepisode photographs $(n=399)$, recent-boundary or terminating-episode photographs $(n=399)$, and photographs within event boundaries $(n=1,721)$. The 798 photographs defining boundaries were further subdivided into 562 photographs used to define only event boundaries and 236 used to define both event and theme boundaries. Of these, 119 were used to define a remote boundary for both an event and theme, and 106 were used to define a recent boundary for both an event and a theme (the remaining 11 photographs defined some combination of recent and remote event and theme boundaries). As in Experiment 1, comparatively few stimuli (photographs) were simultaneously associated with event and theme boundaries, and most event boundaries were marked by changes in event content while themes persisted.

\section{EXPERIMENT 3}

The results of Experiments 1 and 2 are consistent in many respects and, although we argue that they reflect the way episodes are organized in memory into events 
and the way events are indexed to themes, the results could also be interpreted as simply the result of the procedures used. As noted, we specifically avoided making any reference to clustering, grouping, or otherwise combining the stimuli (diary entries and photographs) to define events. We also ensured that the table where the tasks were performed was sufficiently large to allow for every stimulus to be used individually to define an event/theme. Still, the participants may have assumed that we expected them to group the stimuli in some way. One way to examine this issue was to manipulate the experimental conditions and determine whether this influenced the way events are formed. Thus, in Experiment 3 (which again used the photograph technique), participants completed the event- and theme-building task twice. Approximately half the participants were given one third of their photographs and asked to define events and themes, and then given all their photographs and asked to do the tasks again. The task order was reversed for the other participants: They defined events with their full set of photographs first, then with one third of their set. If memory structure, rather than experimental instructions, is responsible for the way events were defined, the number of events/themes defined at each phase (part- vs. whole-stimulus set) should remain reasonably constant, with only the number of photographs used to define the events varying. In contrast, if participants form events on the basis of an assumed expectation of the researchers, they might form events of about the same size in each condition and produce substantially fewer events in the part-stimulus set condition. Furthermore, there should be consistency in the components assigned to the same event under the part- and whole-stimulus set conditions. Slight differences were expected in the number of events defined because both Experiments 1 and 2 showed that a reasonable number of events were defined by a single stimulus, so it was probable that the part stimulus sets would not contain some of these.

\section{Method}

Participants. Fifteen students, 9 females aged 18-30 years $(M=25)$ and 6 males aged $20-34$ years $(M=28.6)$, participated in return for their photographs.

Photography. Participants were given access to digital cameras, which they could sign out whenever they wished over their summer vacation. The Kodak DC50 digital camera takes full-color digital images containing $756 \times 504$ pixels and records the date and time that each image is taken. Participants were instructed to photograph their activities the way they normally would, to take each photograph themselves, and to return the camera to the laboratory for downloading whenever it was full. Each photograph was printed $16 \times 10.5 \mathrm{~cm}$ in full color. Prints that were out of focus or badly exposed were removed. All other photographs were used with no restriction on the number of photographs from a single day. Minimum retention interval was calculated for each participant using the exposure date of his/her last photograph and the date on which the participant completed the experiment. This ranged from 5 to 139 days $(M=64)$.

Procedure. The experiment involved four phases, the order of which was counterbalanced across participants. In two phases, participants were given all of their photographs, asked to define the events they had experienced over the summer, and then asked to define themes in their lives. In a further two phases, participants were given one third of their photographs and asked to do the same two tasks. To provide the participant with one third of his/her photographs, the entire set was placed into temporal order and every third photograph from the set was used to form the part-stimulus set. There was no time limit on any phase, and participants provided labels for the events and themes that they defined at each phase.

\section{Results and Discussion}

A total of 985 photographs were provided by the 15 participants $(M=65$, range $=20-120)$. A total of 72 themes were defined when the task was performed with the whole-stimulus set, and 67 themes were defined when the task was performed with the part-stimulus set. Eleven of the participants defined exactly the same number of themes regardless of whether they performed the task with the part- or the whole-stimulus set. Thus theme identification was influenced very little by the size of the stimulus set with which the participant worked.

The event construction task, when performed with the whole-stimulus set, produced a total of 146 events, 22 $(15 \%)$ of which were defined by a single photograph; when this task was performed with the part-stimulus set it produced a total of 96 events, $17(17.7 \%)$ of which were defined by a single photograph. Five participants defined exactly the same number of events regardless of whether they were using the part- or whole-stimulus set, and a further 2 participants varied by only 1 in the number of events they defined at each phase. As expected, there was a reduction in the number of defined events when the part-stimulus set was used, and some of this reduction can be explained by the unavailability of photographs that were used by themselves to define events (i.e., single-photograph events) in the whole-stimulus set phase.

The labels that the participants provided for their events and themes were used to match up the events and themes defined under the part-stimulus set condition with events and themes defined under the whole-stimulus set condition. Participants were very consistent in their event and theme labeling; it was possible to match $97.9 \%$ of the events and $95.5 \%$ of the themes formed under the partstimulus set condition with an event/theme formed under the whole-stimulus set condition. A perfect event match (i.e., the participant defined exactly the same events under both part- and whole-stimulus set conditions) was obtained for 10 participants, and a perfect theme match was obtained for 11 participants.

Once events and themes were matched, it was possible to determine whether the same photographs had been used to define the same event and theme in the part-stimulus set condition as were used in the whole-stimulus set condition (with the exception that there would be fewer in the part-stimulus set condition). A consistency score was used, where a score of zero indicated that exactly the same photographs had been used to define an event/theme at both the whole- and part-stimulus set phases. Seventyone of the events had a consistency score of zero $(75.5 \%$ 
of the matched events), and the mean event consistency score was 1.1. The mean consistency score for themes was 0.9 , and 48 of the themes had a consistency score of zero ( $75 \%$ of the matched themes). Overall, the matching results and consistency scores indicate that the participants were using essentially the same event and theme representations with their stimuli regardless of the size of the stimulus set they were working with.

\section{GENERAL DISCUSSION}

The process of event construction observed in Experiments 1,2, and 3 raises some interesting questions about what constitutes an autobiographical event. $\mathrm{Nu}-$ merous studies of autobiographical memory have assumed that an experience on a single day represents an autobiographical event (see Conway, 1990, for review). In contrast, our participants often combined a number of on-one-day "episodes" to produce representations that they considered to be autobiographical "events." Indeed, about $70 \%$ of the diary entry and photograph episodes were combined to form events. Clearly, then, what people take to be an autobiographical event is often more complex, extended in time, and consisting of more episodes than the operational version of "events" frequently used in autobiographical memory research.

In addition, Experiments 1 and 2 showed that a large number of event episodes were associated with more than 1 and up to 11 different themes. This interconnectivity of autobiographical knowledge indicates that about half of the "events" sampled in the present study consisted of multiple episodes and multiple themes. Indeed, these complex events might be viewed as a type of theme-nexus in autobiographical memory in which important themes (and their associated goals) become configured in a representation that binds together a set of separate experiences. As an illustration, Figure 1 shows the component episodes (veridical diary entries) that were used to construct one of the events (as labeled by the participant) in Experiment 1 and the themes (as labeled by the participant) to which this event was indexed. Dates are shown for each episode; thus the remote and recent boundaries are identified. The number of other event components that each theme was associated with is also shown.

According to Conway's (1996) and Conway and Pleydell-Pearce's (2000) accounts of autobiographical memory, specific autobiographical memories are constructed from event-specific knowledge, general events, and lifetime periods. Moreover, they proposed that this construction takes place in the conjunction of the working self with the autobiographical knowledge bases (the so-called self-memory system) and occurs when the complex goal system of the working self (defined by Conway \& Pleydell-Pearce as "a subset of working memory control processes organized into interconnected goal hierarchies which functions to constrain cognition"'[p. 265]) modulates knowledge access and memory construction. The present findings suggest that the notion of "episodes" might be usefully introduced to the self-memory system model and that themes, which relate to lifetime periods, might reflect in part the influence of the working self goal system on the autobiographical memory knowledge base. Thus, episodes might be represented in the knowledge base largely by event-specific knowledge (see Conway, 1992; Conway \& Pleydell-Pearce, 2000), and an event might be a representation that binds together sets of event-specific knowledge, perhaps by providing a cue that can be used to access parts of the event-specific knowledge (see Anderson \& Conway, 1993).

A theme, on the other hand, is a much more abstract form of autobiographical knowledge. In the present experiments, themes referred to relationships with partners, family members, friends, and colleagues, and to major life events such as moving house or job (or both), progress in work, and so on. Such themes reflect the main goals of the working self and, according to Conway and Pleydell-Pearce (2000), are one way in which the goal structure of the self becomes represented in autobiographical memory. This is thought to be mainly at the level of lifetime periods, since the goal structure is thought to change extensively only with the occurrence of major life transitions. Events and episodes are representations that provide, as it were, the "data" that ground themes and goals in actual or recorded experience.

Events, then, are important in autobiographical memory because they provide a link from more abstract, thematic, goal-based autobiographical knowledge to representations of specific experience in episodes and event-specific knowledge. It is striking that in Experiments 1 and 2 events were quite sharply delineated by episodes judged strongly associated with recent and remote event boundaries. Possibly, this sharpness of definition attests to the importance of the organizing role of events in autobiographical memory. Also significant here is the finding that events were organized in terms of relations between the content of episodes, and to some extent in terms of themes common to event-related episodes. Interestingly, boundary determination was judged as easier for themes than for events in Experiment 1, but this finding was reversed in Experiment 2. These are small but reliable effects and may indicate that the more detailed event-specific knowledge available in Experiment 2 supported boundary determination better than the less detailed diary entries of Experiment 1. Thus, although events in both experiments were constructed using content association, the fine resolution provided by the photograph technique perhaps made it easier for the participant to identify precisely when a flow of experience moved from one event to another.

A key question is whether our data represent some kind of natural autobiographical memory organization or simply reflect the use of semantic memory through schema activation. Our experiments provide data with relatively short retention intervals, and it is possible that the nature of events, themes, and event/theme relations would change if a substantially longer retention interval had been used. Initially, individuals' experiences are 


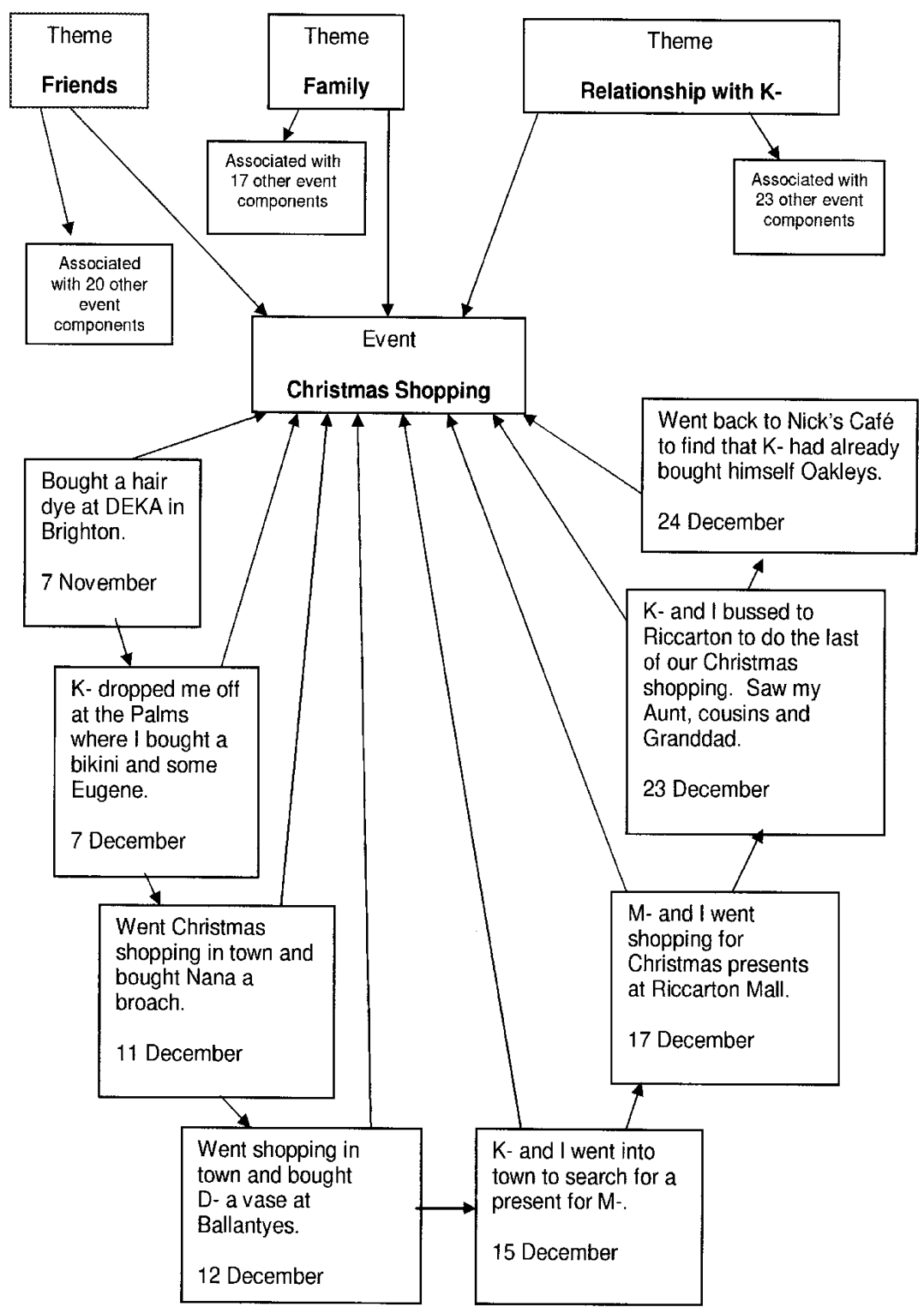

Figure 1. The associative structure for one event from Experiment 1.

clearly autobiographical, defined by extended, complex, and interconnected components (as we found) with numerous links to associated themes. Arguably, access to experiences, and thus the complexity of events, is likely to decrease over time. The loss of event-specific knowledge with time could then result in more schema-based categorization of experiences. Future research could examine this by running the types of experiment we used and requesting the participants to repeat the task after an extended delay. Our prediction is that the number of defined events would increase with time. Experiences that were no longer clearly linked with an event would be clustered using schema knowledge. At the same time, events (like those identified in our experiments) would be formed but the number of component episodes in them would be reduced. The latter prediction is based on the assumption that the boundary components of events will remain accessible - perhaps for decades. Evidence supporting this comes from our work on the estimation of duration of autobiographical events, which showed essentially no change in accuracy over a 10 -year retest interval (Burt, Kemp, \& Conway, 2001).

Should memory researchers stop using on-one-day episodes, such as are collected in diaries, as experimental stimuli? Absolutely not. However, such stimuli may need to be clustered in an initial experimental phase if the researcher is interested in phenomena relating to autobiographical events. If autobiographical events are bigger and more complex than the episodes presented in many experiments, then we may actually be underesti- 
mating performance characteristics. For example, numerous studies have concluded that individuals are relatively poor at dating autobiographical events (e.g., Burt, 1992; Burt et al., 2001; Wagenaar, 1986), but in fact these studies may have examined the dating of episodes from events. It is possible that the bigger, more complex event structures could actually be temporally placed with some degree of accuracy. Each of the complex nested structures of autobiographicalmemory-episodes, events, and themes - can be examined empirically, and future research should clarify what structural aspect of autobiographical memory is under investigation.

\section{REFERENCES}

Anderson, S. J., \& Conway, M. A. (1993). Investigating the structure of autobiographical memories. Journal of Experimental Psychology: Learning, Memory, \& Cognition, 19, 1-19.

Barsalou, L. W. (1988). The content and organization of autobiographical memories. In U. Neisser \& E. Winograd (Eds.), Remembering reconsidered: Ecological and traditional approaches to the study of memory (pp. 193-243). New York: Cambridge University Press.

BURT, C. D. B. (1992). Retrieval characteristics of autobiographical memories: Event and date information. Applied Cognitive Psychology, 6, 389-404.

Burt, C. D. B., Kemp, S., \& Conway, M. A. (2001). What happens if you retest autobiographical memory 10 years on? Memory \& Cognition, 29, 127-136.

Burt, C. D. B., Kemp, S., Grady, J. M., \& Conway, M. (2000). Ordering autobiographical experiences. Memory, 8, 323-332.

Burt, C. D. B., Mitchell, D. A., Raggatt, P. T. F., Jones, C. A., \& Cowan, T. M. (1995). A snapshot of autobiographical memory retrieval characteristics. Applied Cognitive Psychology, 9, 61-74.

Burt, C. D. B., Watt, S. C., Mitchell, D., \& Conway, M. A. (1998). Retrieving the sequence of autobiographical event components. Applied Cognitive Psychology, 12, 321-338.

Conway, M. A. (1990). Autobiographical memory: An introduction. Buckingham, U.K.: Open University Press.

Conway, M. A. (1992). A structural model of autobiographical memory. In M. A. Conway, D. C. Rubin, H. Spinnler, \& W. A. Wagenaar
(Eds.), Theoretical perspectives on autobiographicalmemory (pp. 167194). Dordrecht: Kluwer.

Conway, M. A. (1996). Autobiographical memories and autobiographical knowledge. In D. C. Rubin (Ed.), Remembering our past: Studies in autobiographicalmemory (pp. 67-93). Cambridge: Cambridge University Press.

Conway, M. A., Collins, A. F., Gathercole, S. E., \& Anderson, S. J. (1996). Recollections of true and false autobiographical memories. Journal of Experimental Psychology: General, 125, 69-95.

Conway, M. A., \& Pleydell-Pearce, C. W. (2000). The construction of autobiographical memories in the self-memory system. Psychological Review, 107, 261-288.

Friedman, J. W. (1993). Memory for the time of past events. Psychological Bulletin, 113, 44-66.

Haque, S., \& Conway, M. A. (2001). Probing the process of autobiographical memory retrieval. European Journal of Cognitive Psychology, 13, 529-554.

Linton, M. (1975). Memory for real world events. In D. A. Norman \& D. E. Rumelhart (Eds.), Explorations in cognition (pp. 376-404). San Francisco: Freeman.

NEISSER, U. (1986). Nested structure in autobiographical memory. In D. C. Rubin (Ed.), Autobiographical memory (pp. 71-81). Cambridge: Cambridge University Press.

NEISSER, U. (1988). What is ordinary memory the memory of? In U. Neisser \& E. Winograd (Eds.), Remembering reconsidered: Ecological and traditional approaches to the study of memory (pp. 356373). New York: Cambridge University Press.

Robinson, J. A. (1992). First experience memories: Contexts and function in personal histories. In M. A. Conway, D. C. Rubin, H. Spinnler, \& W. A. Wagenaar (Eds.), Theoretical perspectives on autobiographical memory (pp. 223-239). Dordrecht: Kluwer.

Shum, M. S. (1998). The role of temporal landmarks in autobiographical memory processes. Psychological Bulletin, 124, 423-442.

WagenaAR, W. A. (1986). My memory: A study of autobiographical memory over 6 years. Cognitive Psychology, 18, 225-252.

Wheeler, M. A., Stuss, D. T., \& Tulving, E. (1997). Toward a theory of episodic memory: The frontal lobes and autonoetic consciousness. Psychological Bulletin, 121, 351-354.

White, R. T. (1982). Memory for personal events. Human Learning, 1, 177-183.

(Original manuscript received March 26, 2002; revision accepted for publication October 3, 2002.) 